\title{
Cosmic Rays from Direct Measurements and from Multifrequency Observations of Interstellar Emissions
}

\section{Elena Orlando ${ }^{1}$}

Kavli Institute for Particle Astrophysics and Cosmology and Hansen Experimental Physics Laboratory, Stanford University, CA (USA)

E-mail: orlandele@gmail.com

Interactions of cosmic rays with the interstellar medium and Galactic magnetic fields produce diffuse emissions from radio and microwaves, to gamma-ray energies. Observations of these diffuse emissions are an invaluable tool for understanding densities and spectra of cosmic rays in different places of our Galaxy.

We present local interstellar cosmic-ray spectra, independent of solar modulation, and propagation properties for the first time derived by combining the latest cosmic-ray direct measurements with multi-frequency data of the interstellar emissions observed with radio surveys, Planck, and Fermi Large Area Telescope.

This paper discusses the results based on the work published in Orlando (2018) MNRAS 475, 2724. Details are reported in that paper.

36th International Cosmic Ray Conference -ICRC2019-

July 24th - August 1st, 2019

Madison, WI, U.S.A.

\section{${ }^{1}$ Speaker}


Cosmic Rays from Direct Measurements and from Multifrequency Observations of Interstellar Emissions

This paper discusses the results based on the work published in Orlando (2018) MNRAS 475, 2724. Details are reported in that paper. 